\title{
GAUSSIAN NOISE REDUCTION ON IMAGES AUTOMATICALLY
}

\author{
Meera Devi R V ${ }^{1}$, Sathish Kumar B $\mathbf{S}^{2}$ \\ ${ }^{1}$ M.E scholar, A.V.C College of Engineering, Mannampandal, Mayiladuthurai, India \\ ${ }^{2}$ Associate Professor, A.V.C College of Engineering, Mannampandal, Mayiladuthurai, India
}

\begin{abstract}
The various images can be taken by camera, due to some artifacts image become noisy and blur. As a result the image may be containing the noise and unwanted details. This noise will be degrading the image information. The proposed method also used removes the noise in images. These methods are using combined total variation and wavelet thresholding to removing the noise from images. The image quality can be measured using the MSE (Mean Square Error),PSNR (Peak Signal to Noise Ratio),Entropy, Correlation Coefficient.
\end{abstract}

Keywords: Noise, Total variation, PSNR, MSE.

\section{INTRODUCTION}

Image processing is widely used in real world, so it needs lot of algorithm for developing this field. The image processing image denoising is field to remove the noise in images. The image noise are classified to Speckle, Gaussian ,Poisson, and salt and pepper noise[1]. The speckle noise is also called multiplicative noise. The Gaussian noise is also called additive noise. The salt and pepper noise is also called black and white noise[2]. The noise are degrading the image quality and information.

The section 2 discussing the literature review, section 3 describing the existing method, section 4 explained the proposed method and section 5 discussing the results and discussion and section 6 conclusion of the paper.

\section{LITERATURE REVIEW}

Image denoising is also called image restoration techniques [3].The image processing various filtering methods. The filtering methods are used for image enhancement and denoising. The filters are mean, median, and Wiener filters. The median filter it would be removing noise in image, but the median filter it depends on mask size chosen. The mask size is larger the image become blur and over smoothing. The mean filter is an averaging filter, which is not suitable Gaussian noise[4].The wiener filter being used for image restoration and also having same drawback as median.

The image transforms are FFT(Fast Fourier transform), DCT(Discrete Cosine Transform), Radon transform, Dual tree complex wavelet transform, wavelet transform. The FFT, DCT and radon transform are not suitable for Gaussian noise[5].The dual tree complex wavelet transform having the high computational complexity is high compared with wavelet[6].The wavelet transform is used.

There are iterative algorithms also used for image denoising. There are landweber, Total variation and blind deconvolution. The methods are resulting good output , but it produce moderated quality measure[7]. The major problem in image denoising performing these above algorithm results edge information and other details.

\section{EXISTING SYSTEM}

The existing system has using wavelet thresholding method to removing the noise in image, yet this wavelet transform gives moderate quality. Curevelet transform also used in image denoising but it computation time is high. The proposed method is combined the iterative techniques total variation and wavelet thresholding. The proposed method improves image result and without loss of edge information.

\section{PROPOSED SYSTEM}

The discrete wavelet transform is used for image noise removal. It will perform the sub band decomposition. This decomposition is done by row and column wise processing the image. The wavelet transform single level decomposition diagram is shown below(figure1).

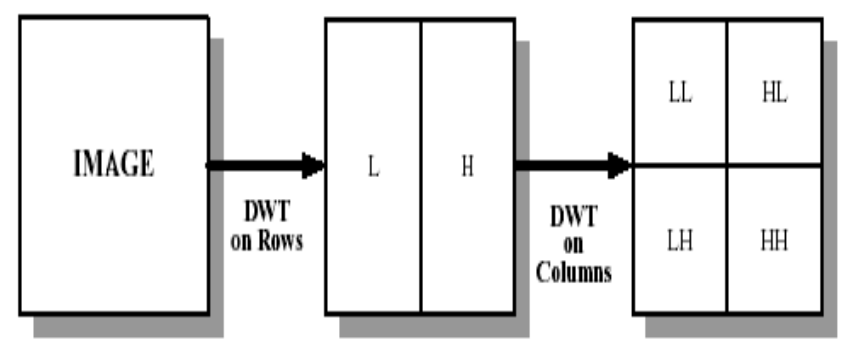

Fig 1: DWT structure

Wavelet split image into 4 sub bands such as LL, LH, HL, $\mathrm{HH}$.low pass perform Averaging operation extract the coarse information of the image. The high pass corresponds to differencing operation; extract the detail information of the signal. The output of filtering operation is decimated by 2. The Sub band (LL, LH, HL,HH) are the Filter coefficients of the sub band methods. That are the horizontal vertical and diagonal pixel values of the Images. LL: Vertical Low pass \& Horizontal Low pass, LH: Vertical Low pass\& Horizontal High pass, HL: Vertical High pass\& Horizontal Low pass, HH: Vertical High pass \& Horizontal High pass. 
The proposed block diagram is shown below (figure2). The first block image can be taken in files. The second block it can converting into gray image. The noise is adding in the image. Then apply the DWT and getting subbands. The subbands apply total variation and wavelet thresholding was performed. IDWT (inverse wavelet transform ) to combine the subbands and again apply total variation and get the denoise output.

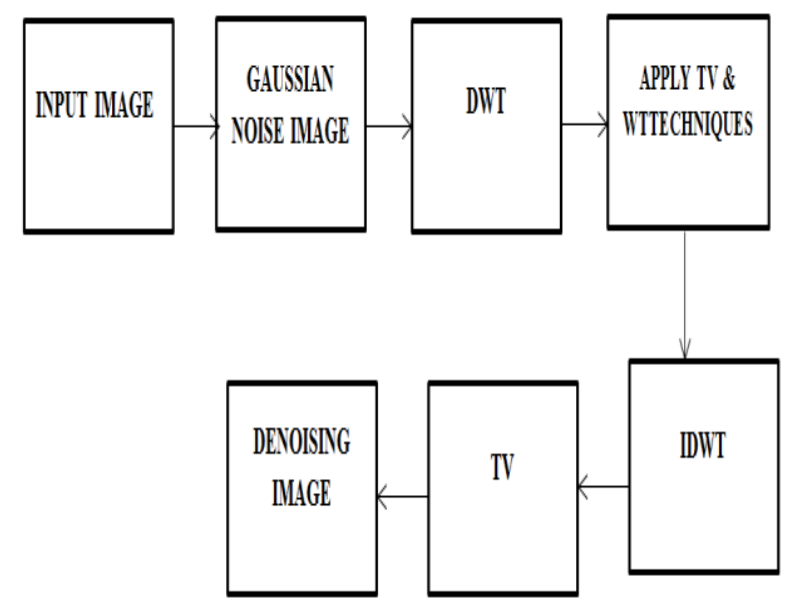

Fig 2: Block diagram

\subsection{Total Variation}

The total variation is one of the iterative techniques to enhance image. the difference between two pixels calculated .if it higher than is noisy pixel to remove it. The total variation is a method for noise reduction, it can be applied for LL and IDWT output. The total variation is a method removing the high variance pixel in the image. Placing normal variance pixel as it. The threshold variance value is set to remove high variance pixel. These results the good quality.

\subsection{Wavelet Thresholding}

Wavelet Thresholding (first proposed by Donoho) is a signal estimation technique that exploits the capabilities of wavelet transform for image denoising. It removes noise by reducing coefficients that are insignificant relative to some threshold. The wavelet Thresholding, the methods are given below.

- Universal Thresholding

- Visu Shrink

- Sure Shrink

- $\quad$ Bayes Shrink

Here using the Soft Thresholding to remove the noise. The Global threshold is well known in threshold.

$$
\lambda_{U N I V}=\sqrt{2 \ln N} \sigma
$$

The thresholding operator for soft is given by

$$
\mathrm{D}(\mathrm{U}, \lambda)=\operatorname{sgn}(\mathrm{U}) \max (0,|\mathrm{U}|-\lambda)
$$

Soft Thresholding less the coefficients above the threshold in absolute value. These wavelet thresholding method is applicable for LH, HL, HH remaining sub bands.

\section{PERFORMANCE AND RESULTS}

The performance metrics measure the image quality .The performance metrics are MSE, PSNR, Entropy and correlation coefficient.

$$
\mathrm{MSE}=\frac{1}{\mathrm{MN}} \sum_{\mathrm{y}=1}^{\mathrm{M}} \sum_{\mathrm{x}=1}^{\mathrm{N}}\left[\mathrm{I}(\mathrm{x}, \mathrm{y})-\mathrm{I}^{\prime}(\mathrm{x}, \mathrm{y})\right]^{2}
$$

$\mathrm{PSNR}=20 * \log 10\left(255^{\wedge} 2 /(\mathrm{MSE})\right)$

MSE is calculated as per the above formula (3) Calculating the sum of and difference of original and output image and divided by image size .PSNR is measure peak error in images. It is measured in decibels and take the log of pixel value and divided by MSE.

Where $I(x, y)$ is the original image, $I^{\prime}(x, y)$ is the approximated version (which is actually the decompressed image) and M,N are the dimensions of the images. A good quality image low MSE and High PSNR. Correlation coefficient is to measure the similarities between two images. Entropy is measuring information about the content source. 


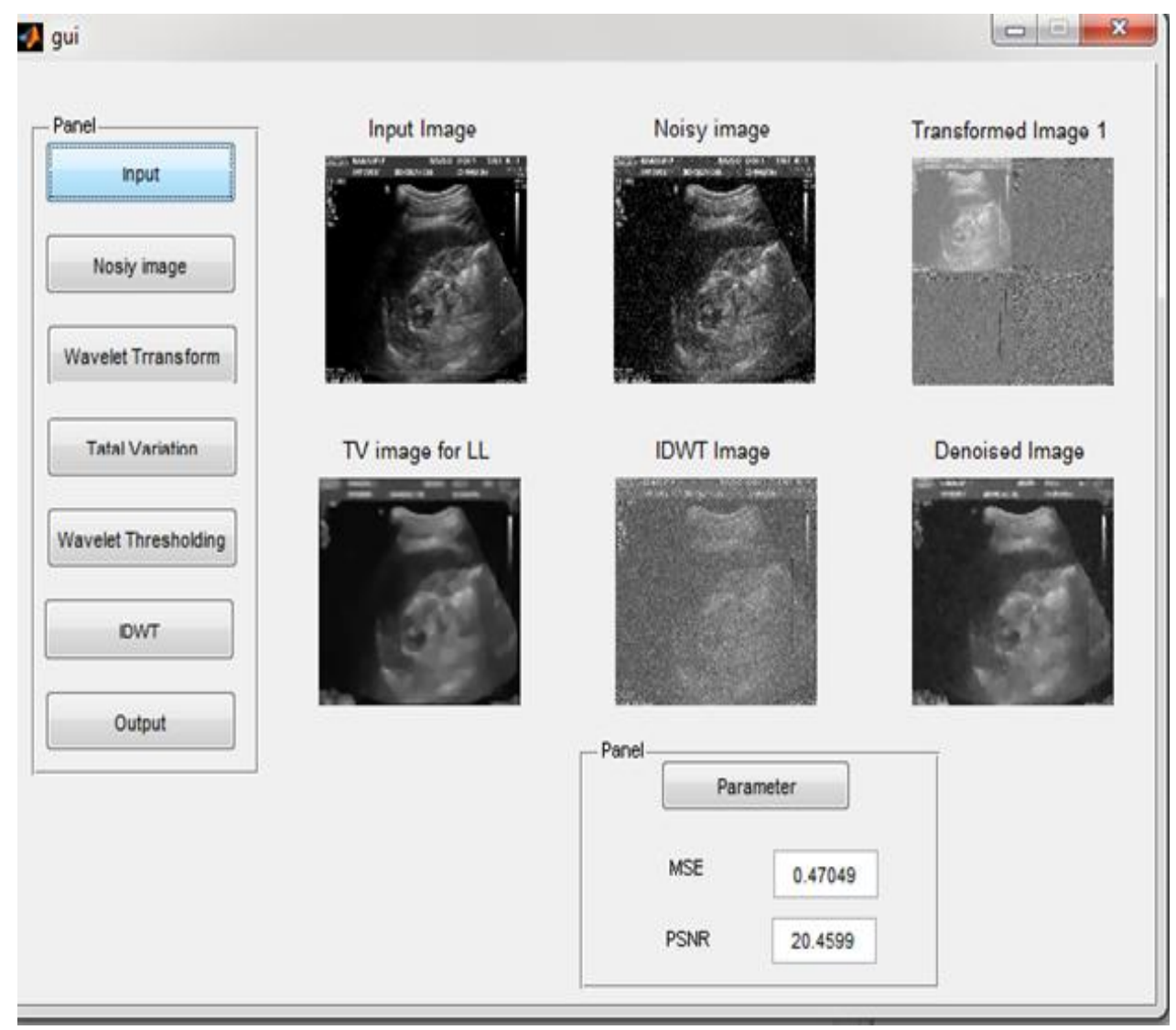

Fig 3: Medical Image Result

These algorithm implemented in MATLAB 7.10 version using GUI(Graphical user interface) .The result performance analysis in three different images. The medical image result is shown figure3.

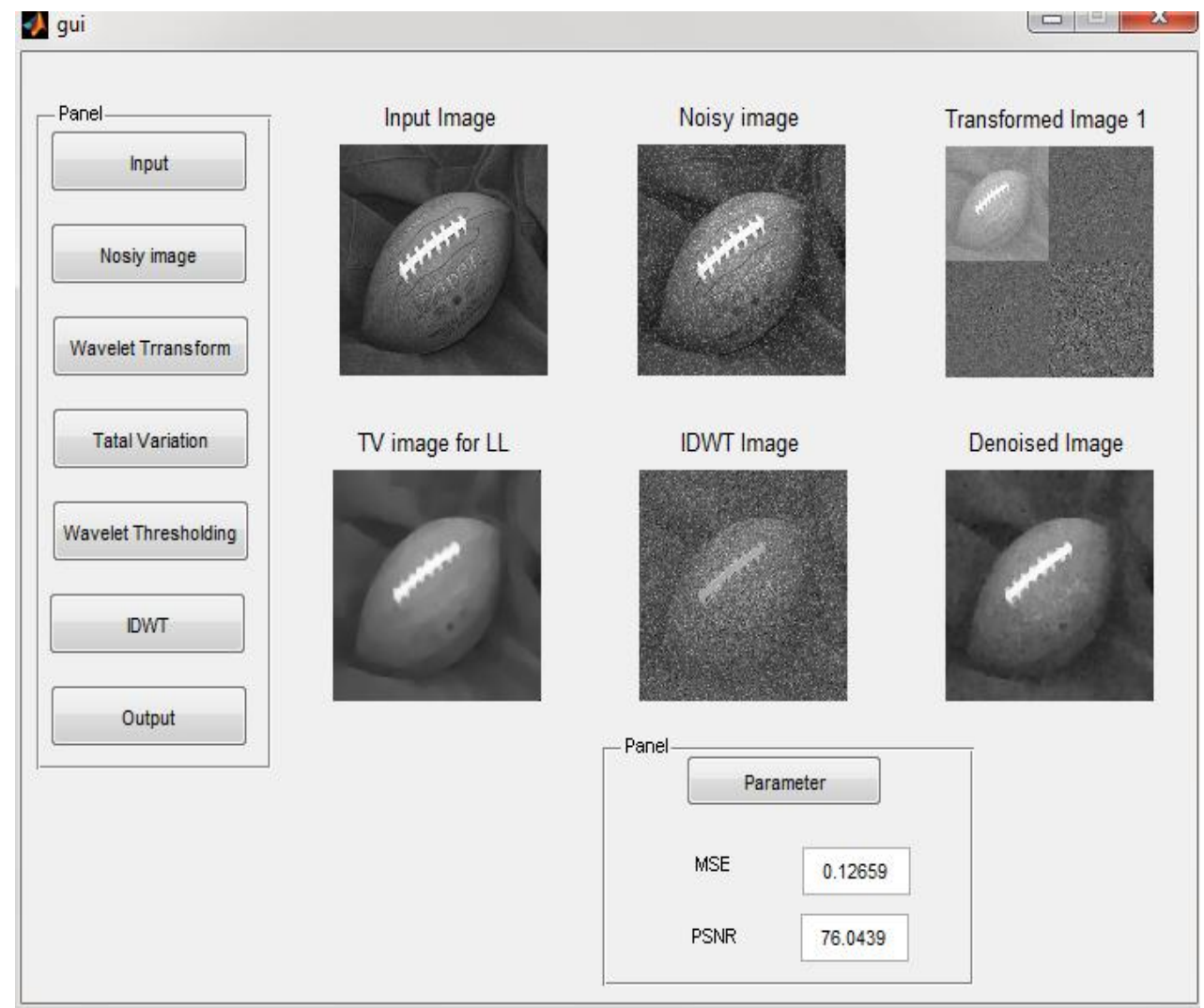

Fig 4: Football Image Result 
The football image result is shown above figure 4. The third result performance image is rice image.. The result shown in figure 5.Then the MSE, PSNR, given in table1.

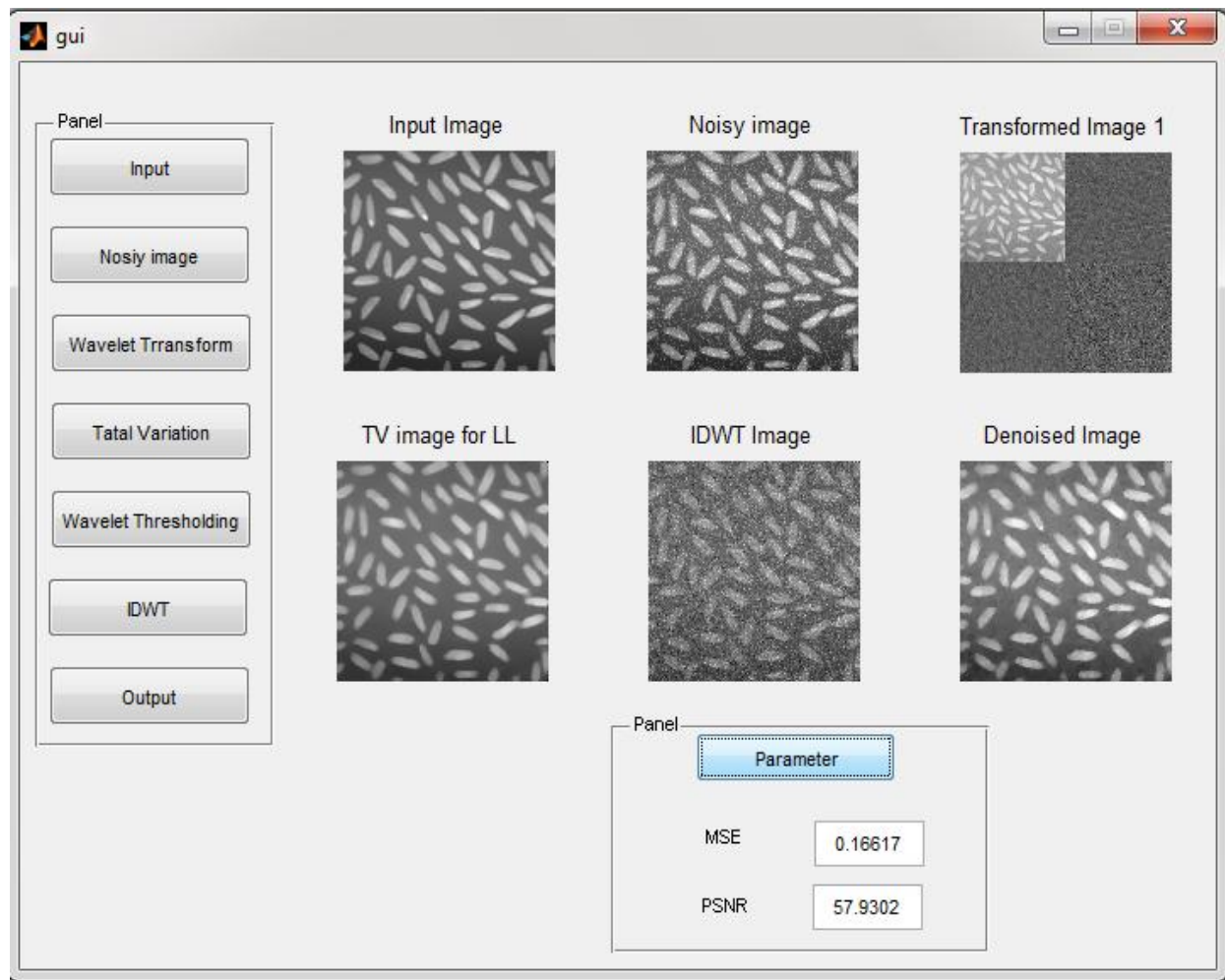

Fig 5: Rice Image Result

Table: 1 Performance metrics of MSE and PSNR

\begin{tabular}{|l|l|l|l|}
\hline S.NO & IMAGE NAME & MSE & PSNR \\
\hline 1 & MEDICAL & 0.47049 & 20.4599 \\
\hline 2 & FOOT BALL & 0.12659 & 76.0439 \\
\hline 3 & RICE & 0.1667 & 57.9302 \\
\hline
\end{tabular}

Entropy, and correlation coefficient is given in table2.

Table: 2 Performance metrics of correlation coefficient and Entropy

\begin{tabular}{|l|l|l|l|}
\hline S.No & Image Name & Correlation coefficient & entropy \\
\hline 1 & MEDICAL & 5.7933 & 0.9138 \\
\hline 2 & FOOTBALL & 5.5500 & 0.9588 \\
\hline 3 & RICE & 6.0047 & 0.9654 \\
\hline
\end{tabular}

\section{CONCLUSION}

The proposed system is reduction on Gaussian additive noise in ultrasound image. Noise reduction is important in diagnostic purpose because we get the actual information from the images. Using this proposed method we get original image without noise and damage the edges and texture. The obtained denoised CT images are of good quality, which can be utilized for effective and precise disease diagnosis.

\section{REFERENCES}

[1] Yang Wang and Haomin Zhou, "Total Variation Wave Based Medical Image Denoising", International Journal of Biomedical Imaging, Vol. 2006, pp.1-6, January 2006

[2] Sudha, Suresh and Sukanesh, "Comparative Study on Speckle Noise Suppression Techniques for Ultrasound Images", International Journal of Engineering and Technology, Vol. 1, No. 1, pp. 5762, April 2009

[3] Hyder Ali, Sukanesh and Fellow, "An Edge Preserving Denoising Technique for MR Images using Curvelet Transform" ,Interdisciplinary Journal, Vol. 91, pp. 3-8, May 2010

[4] Shujun Fu, Qiuqi Ruan, Wenqia Wang and $\mathrm{Yu} \mathrm{Li}$, "Adaptive Anisotropic Diffusion for Ultrasonic Image Denoising and Edge Enhancement", International Journal of Information Technology, Vol. 2, No. 4, pp. 284-287, 2006

[5] Shujun Fu, Qiuqi Ruan, Wenqia Wang and Yu Li, "Feature Preserving Nonlinear Diffusion for Ultrasonic Image Denoising and Edge Enhancement",WorldAcademy of Science, Engineering and Technology, Vol. 2, pp. 148-151, February 2005

[6] Tanaphol Thaipanich and Jay Kuo, "An Adaptive Nonlocal Means Scheme for Medical Image Denoising", In Proceedings of SPIE Medical Imaging, Vol. 7623, San Diego, CA, USA, February 2010 
[7] Su Cheol Kang and Seung Hong Hong, "A Speckle Reduction Filter using Wavelet- Based Methods for Medical Imaging Application", In Proceedings of 23rd Annual International Conference of the IEEE Engineering in Medicine and Biology Society, Vol. 3, pp. 2480-2483,

[8] Luis Marti-Bonmati and Montserrat Robles, "Multicomponent MR Image Denoising", Journal of Biomedical Imaging, Vol. 2009, No. 18, pp. 1-27, 2009

[9] Perona and Malik, "Scale-space and edge detection using anisotropic diffusion", IEEE Transaction on Pattern Analysis and Machine Intelligence, Vol. 12, No. 7, pp. 629-639, 1990

[10] Manjon, Robles and Thacker, "Multispectral MRI de-noising using non-local means", In Proceedings of MIUA, pp. 41-46,

[11] Gerig, Kubler, Kikinis and Jolesz, "Nonlinear Anisotropic Filtering of MRI Data", IEEE Transaction on Medical Imaging, Vol. 11, No. 1, pp. 221-232, 1992

\section{BIOGRAPHIES:}

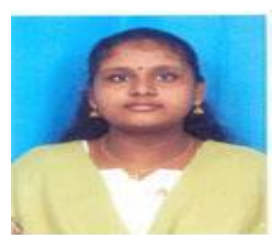

R.V.Meeradevi Pursuing M.E Applied Electronics in AVC college of engineering. She obtained her $\mathrm{BE}$ degree in A.V.C. College of Engineering,Anna University chennai.she has published natinal and international journal.Her area of interest in Image Denoising ,Image Enhancement ,Embedded System.

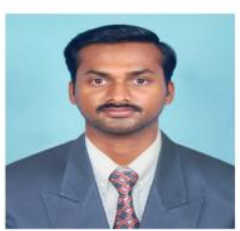

Mr.Sathish Kumar doing his Ph.D degree in Pondicherry University. He Completed his PG Degree in 2002 from SASTRA University and UG degree in 2001 from Bharadhidasan university. He is also the member in ISTE and BME. He published a Paper in International and national Journal and Conference. His area of interest in image processing and communication. 